\title{
EFEITOS DA TERAPIA MANUAL NA REABILITAÇÃO DA SÍNDROME DO IMPACTO DO OMBRO: REVISÃO BIBLIOGRÁFICA
}

EFFECTS OF MANUAL THERAPY IN REHABILITATION OF SHOULDER IMPINGEMENT SYNDROME: LITERATURE REVIEW

\section{Lorrany Terezinha Oliveira de Souza}

Acadêmica do curso de Fisioterapia da Faculdade Evangélica de Ceres, Ceres- Go, Brasil.

E-mail: lorranyninha@gmail.com

\section{Mateus Silva Camargo}

Acadêmico do curso de Fisioterapia da Faculdade Evangélica de Ceres, Ceres- Go, Brasil. E-mail: mateus-silvacamargo@hotmail.com

\section{Rafael Muryllo Tavares Venâncio}

Acadêmico do curso de Fisioterapia da Faculdade Evangélica de Ceres, Ceres- Go, Brasil. E-mail: rafaelmuryllo16@gmail.com

\section{Rosiane Siriana de Jesus}

Acadêmica do curso de Fisioterapia da Faculdade Evangélica de Ceres, Ceres- Go, Brasil. E-mail: rosisiriana@gmail.com

\section{Endereço para correspondência:}

Rua MV5, Q.7, L.4, Morada Verde, Ceres-Go, Brasil. CEP: 76300-000

Telefone: (62) 996913431

E-mail: lorranyninha@gmail.com 


\section{RESUMO}

Introdução: A síndrome do impacto do ombro é definida como uma disfunção orgânica do manguito rotador por meio de microtraumas nos tecidos localizados no espaço subacromial resultando em uma dificuldade para executar sua função. A fisioterapia tem grande relevância na sua reabilitação, visando diminuir a inflamação e dor, fortalecer a musculatura, restaurar a amplitude articular, funcionalidade e independência do indivíduo. Objetivo: Analisar a literatura acerca dos efeitos das técnicas de terapia manual na síndrome do impacto do ombro. Metodologia: Trata-se de uma revisão narrativa da literatura com buscas de artigos científicos publicados entre os anos de 2014 a 2019, sem restrição de idioma, indexados nas bases de dados eletrônicas: Pubmed (United States National Library of Medicine), CENTRAL (Cochrane Controlled Register of Trials), BVS (Biblioteca Virtual em Saúde), PEDro (Physiotherapy Evidence Database) e Biblioteca Virtual da Faculdade Evangélica de Ceres. Resultados e discussão: Foram incluídos na revisão 5 artigos e 2 livros, que relataram os efeitos da terapia manual geral e de técnicas específicas como mobilização com movimento no ombro, SNAGs (deslizamentos apofisários sustentados naturais) e Maitland. Conclusão: A terapia manual mostrou ser eficaz na reabilitação da síndrome do impacto do ombro, apresentando diminuição da dor, aumento da força muscular, melhora da amplitude de movimento e da capacidade funcional do ombro. A técnica de mobilização com movimento mostrou maior efetividade quando aplicada diretamente ao ombro, porém SNAGs e Maitland apresentaram significativa eficácia quando aplicadas a coluna torácica.

Palavra-chave: Síndrome do Impacto. Ombro. Terapia Manual. Reabilitação. 


\begin{abstract}
Introduction: The Shoulder impingement syndrome is defined as a dysfunction organic of the rotator cuff by means of microtraumas in tissues located in the subacromial space resulting in a difficulty to perform its function. Physiotherapy has great relevance in the rehabilitation of the syndrome, aiming to decrease inflammation and pain, strengthen muscle, restore joint amplitude and restore, functionality and independence of the individual. Objective: To analyze the literature on the effects of manual therapy techniques as rehabilitation of shoulder impingement syndrome. Methodology: This is a narrative review of the literature based on searches of scientific articles published between the years 2014 to 2019, without restriction of language, indexed in the electronic databases: Pubmed (United States National Library of Medicine), CENTRAL(Cochrane Controlled Register of Trials), BVS (Biblioteca Virtual em Saúde), PEDro (Physiotherapy Evidence Database) and searches of books in the Virtual Library of College Evangelical of Ceres. Results and discussion: Were included in the review 5 articles and 2 books, what reported the efficacy of general manual therapy and specific techniques such as mobilization with shoulder movement, SNAGs (Sustained natural apophyseal glides) and Maitland thoracic. Conclusion: Manual therapy has been shown to be effective in the rehabilitation of the shoulder impingement syndrome, with reduced pain, increased muscle strength, improved range of motion and functional capacity of the shoulder. The movement mobilization technique showed greater effectiveness when applied directly to the shoulder, but SNAGs and Maitland presented significant efficacy when applied to the thoracic spine.
\end{abstract}

Keywords: Impingement Syndrome. Shoulder. Manual Therapy. Rehabilitation. 


\section{INTRODUÇÃO}

A síndrome do impacto do ombro (SIO) foi inicialmente descrita por Neer (1972), que a definiu como uma disfunção orgânica do manguito rotador por meio de microtraumas nos tecidos localizados no espaço subacromial resultando em uma dificuldade para executar sua função fisiológica e a classificou em três graus de gravidade baseado nos critérios fisiopatológicos e clínicos, sendo o grau I caracterizado por inflamação, hemorragia e edema no manguito rotador e bursa subacromial, o grau II definido por alterações irreversíveis e o grau III apresentando mudanças crônicas.

A síndrome do impacto no ombro se caracteriza por impactação mecânica de estruturas que se localizam no espaço subacromial e ocorre pelo estresse do manguito rotador devido a repetição do movimento sob o arco coracoacromial ou quando o acrômio, o ligamento coracoacromial ou o processo coracóide invadem a superfície do manguito rotador durante a abdução acima de 90 graus, diminuindo o espaço subacromial e resultando em uma compressão principalmente do tendão do músculo supraespinhoso ou até mesmo do tendão da cabeça longa do bíceps, da bursa subacromial ou do tendão do infraespinhoso (METZKER, 2010).

A intervenção fisioterapêutica tem como por objetivos gerais ocasionar um alívio do quadro álgico, gerar aumento da força muscular e amplitude de movimento, de maneira a obter uma melhora da funcionalidade do membro acometido. As técnicas de terapia manual objetivam diminuir a inflamação subacromial, para proporcionar a uma cicatrização e o fortalecimento da musculatura estabilizadora para restaurar a função (SENBURSA; BALTACI; ATAY, 2007). Diante disso, o presente estudo é baseado na seguinte problemática: As técnicas de terapia manual são eficazes na reabilitação da síndrome do impacto do ombro?

A relevância desse trabalho se justifica pelas incapacidades ocasionadas pela SIO no qual é caracterizada pela presença de dor intensa na região anteroposterior e lateral da articulação, necessitando de técnicas específicas que visem diminuir os sintomas, incapacidades e a reabilitação do complexo do ombro.

Portanto, o objetivo do presente estudo foi analisar a literatura acerca dos efeitos das técnicas de terapia manual como reabilitação na síndrome do impacto do ombro.

\section{METODOLOGIA}


O presente estudo trata-se de uma revisão narrativa da literatura. Foram realizadas buscas de artigos científicos publicados entre os anos de 2014 a 2019, sem restrição de idioma, indexados nas bases de dados eletrônicas: Medline/ Pubmed, Embase, CENTRAL, Lilacs/BVS e PEDro, utilizando os descritores: impingement syndrome, manual therapy, rehabilitation e shoulder, adaptados ao idioma de acordo com as bases de dados e combinados pelos operadores booleanos AND e OU. Buscas também foram realizadas na Biblioteca Virtual da Faculdade Evangélica de Ceres afim de identificar livros publicados sobre a temática sem restrição de data de publicação.

Os livros e artigos científicos foram pesquisados e analisados durante o período entre março a abril de 2017, obedecendo a alguns critérios de inclusão e exclusão, sendo que os critérios de inclusão definidos foram: artigos com intervenção de terapia manual e estudos com participantes diagnosticados com síndrome do impacto do ombro e como critérios de exclusão: artigos que comparem ou associem a terapia manual com terapia farmacológica; estudos realizados em animais; participantes com patologia associada no ombro e participantes com intervenção cirúrgica à patologia em estudo.

\section{RESULTADOS E DISCUSSÃO}

Foram encontrados 25 artigos e 2 livros inicialmente, após a leitura dos resumos e sumários foram excluídos 20 artigos que não se enquadravam nos critérios de inclusão preestabelecidos. Sendo assim, foram incluídos na revisão 5 artigos e 2 livros que abordaram técnicas de terapia manual para reabilitação da síndrome do impacto do Ombro.

A mobilização em geral contribui no tratamento da SIO para a diminuição da dor através do estímulo mecânico que proporciona respostas neurofisiológicas no sistema nervoso periférico. A mobilização pode ser ainda mais eficaz quando associada com a tração das articulações do complexo do ombro aplicada em todos os eixos de movimento com a graduação da intensidade de acordo com as condições do paciente. Porém é fundamental, antes da aplicação da técnica, avaliar as contraindicações como hipermobilidade articular, estresse prematuro das estruturas, tumores, infecção e inflamação aguda (SOUZA, 2001).

A terapia manual dispõe de diversas técnicas eficazes no processo de reabilitação do ombro. Dutton (2010) descreveu algumas técnicas de terapia manual que podem ser aplicadas ao complexo do ombro: O conceito Cyriax inclui estratégia de tratamento como mobilização, manipulação, massagem friccional e educação do paciente; o conceito Mennell utiliza 
manipulação, educação do paciente e mobilização; o conceito de Kaltenborn inclui técnicas de tração/distração, manipulação, mobilização de tecidos moles e educação do paciente e o conceito de Maitland que preconiza o comprometimento com o paciente a partir da aplicação do pensamento teórico e clínico, avaliação contínua e reavaliação, enfatizando o tratamento da rigidez e da dor com tração, manipulação, exercícios com movimentos, mobilização neural e educação do paciente.

Em um estudo realizado por Camargo et al. (2015) afim avaliar os efeitos de um protocolo de exercícios de força muscular e alongamento comparando com um plano de intervenções de terapia manual realizado durante 4 semanas, com sessões de 45 minutos, no qual as técnicas de terapia manual foram aplicadas apenas no lado afetado dos pacientes. Os resultados mostraram que a inclusão de terapia manual a um protocolo de exercícios não fornece benefícios complementares para melhora da dor e a função. $\mathrm{Na}$ maior parte dos resultados, o grupo de exercício sozinho demonstrou maior benefício do que o grupo de exercício mais terapia manual.

Em contrapartida, evidências de efeitos positivos com técnicas específicas de terapia manual foram encontras. Com o objetivo de comparar os efeitos imediatos da mobilização com o movimento com uma técnica simulada por placebo sobre a amplitude de movimento e dor em pacientes com síndrome do impacto do ombro, Delgado-Gil e colaboradores (2015) realizaram um ensaio clínico randomizado com 42 pacientes e as medidas de resultados primários, incluindo a dor durante a amplitude de movimento ativa, a intensidade da dor e a amplitude de movimento ativa máxima foram avaliadas. Os autores concluíram que os pacientes que receberam a técnica de mobilização com movimento exibiram resultados significativamente melhores para a amplitude de flexão do ombro, melhora da rotação externa máxima e diminuição de dor do que os pacientes que receberam a técnica simulado por placebo.

A terapia manual para reabilitação da SIO demonstra enfatizar principalmente a mobilização articular glenoumeral, porém outras articulações da coluna cervical e torácica podem influenciar na reabilitação. Andrews e colaboradores (2018) investigaram o uso de deslizamentos apofisários naturais sustentados- SNAGs (conceito Mulligan) na coluna torácica para o tratamento do SIO. Como resultado encontraram uma diminuição na dor durante a abdução ativa imediatamente após o tratamento e uma melhora nos escores estatisticamente importantes para rotação externa resistida e abdução ativa após 48 horas de intervenção. Os autores concluíram que os SNAGs torácicos podem influenciar os níveis de dor a curto prazo e mobilidade do ombro nos indivíduos com SIO. Tais resultados podem 
apoiar o conceito de interdependência regional entre a coluna torácica e a articulação glenoumeral.

Haider e colaboradores (2018) compararam o efeito da terapia conservadora com e sem terapia manipulativa torácica de Maitland em 40 pacientes com SIO através de um ensaio clínico randomizado controlado e concluíram que a terapia manipulativa torácica de Maitland com a terapia conservadora foi mais eficaz na redução da dor e melhora do status funcional do ombro do que a terapia conservadora isoladamente.

Em desacordo com os estudos anteriores, Cook e colaboradores (2014) em um ensaio clínico randomizado simples cego com 68 pacientes, não encontraram diferenças significativas entre indivíduos que receberam tratamento baseado em evidências para o ombro isoladamente e indivíduos que receberam terapia manual para cervical adicionada ao tratamento, o que contraria a hipótese da influência da manipulação da coluna cervical para reabilitação do ombro.

\section{CONCLUSÃO}

A terapia manual em geral mostrou ser eficaz na reabilitação da síndrome do impacto do ombro, apresentando diminuição da dor, aumento da força muscular, melhora da amplitude de movimento e da capacidade funcional do ombro. A técnica de mobilização com movimento mostrou maior efetividade quando aplicada diretamente ao ombro, porém SNAGs e terapia manipulativa de Maitland apresentaram significativa eficácia no tratamento da síndrome do impacto do ombro quando aplicadas a coluna torácica. As técnicas de terapia manual identificadas são distintas, sendo assim, cabe ao profissional fisioterapeuta determinar sua aplicabilidade fundamentando-se nas características clínicas particularidades apresentadas pelo paciente.

\section{REFERÊNCIAS}

ANDREWS, D. P. et al. The utilization of mulligan concept thoracic sustained natural apophyseal glides on patients classified with secondary impingement syndrome: a multi-site case series. Int. J. Sports Phys. Ther., v.13, n. 1, p. 121-130, feb. 2018.

CAMARGO, R. P. et al. Effects of Stretching and Strengthening Exercises, With and Without Manual Therapy, on Scapular Kinematics, Function, and Pain in Individuals With Shoulder 
Impingement: A Randomized Controlled Trial. J. Orthop. Sports Phys. Ther., v. 45, n. 12, p.984-997, dec. 2015.

COOK, C. et al. The addition of cervical unilateral posterior-anterior mobilisation in the treatment of patients with shoulder impingement syndrome: a randomised clinical trial. Man. Ther., v. 19, n. 1, p. 18-24, feb. 2014.

DELGADO-GIL, J. A. et al. Effects of mobilization with movement on pain and range of motion in patients with unilateral shoulder impingement syndrome: A randomized controlled trial. J. Manipulative and Phys. Ther., v. 38, n. 4, p. 245-252, 2015.

DUTTON, M. Fisioterapia ortopédica: exame, avaliação e intervenção. 2. ed. Porto Alegre: Artmed, 2010.

HAIDER, R. et al. Comparison of conservative exercise therapy with and without Maitland Thoracic Manipulative therapy in patients with subacromial pain: Clinical trial. J. Pak. Med. Assoc., v. 68, n. 3. p. 381-387, mar. 2018.

METZKER, C. A. B. Tratamento conservador na síndrome do impacto no ombro. Fisioter. Mov., v. 23, n. 1, p. 141-151, jan./mar. 2010.

NEER, C. S. Anterior acromioplasty for the chronic impingement syndrome in the shoulder: a preliminary report. The Journal Bone and joint surgery American, v. 54-A, n. 1, jan. 1972.

SENBRUSA, G.; BALTACI, G.; ATAY, A. Comparison of conservative treatment with and without manual physical therapy for patients with shoulder impingement syndrome: a prospective, randomized clinical trial. Knee Surg Sports Traumatol Arthros. v. 12, p. 951921, feb. 2007.

SOUZA, M. Z. Reabilitação do complexo do ombro. 1. ed. São Paulo: Manole, 2001. 